\title{
Pain Relief Following Radio Frequency Energy Chondroplasty for Isolated Chondral Lesions of the Knee
}

\author{
Timothy Woodacre*, Anna Thomas and Vipul I Mandalia
}

Royal Devon and Exeter Hospital, Exeter, Devon, England, UK

\begin{abstract}
Purpose: We assessed the pain relief achieved by patients following radio frequency energy chondroplasty (RFC) for isolated chondral lesions in the knee.

Methods: Retrospective analysis was completed of operative notes and arthroscopic images of all patients who underwent arthroscopic chondroplasty at the Royal Devon and Exeter Hospital between January 2009 and June 2012. Inclusion criteria included 1 to 2 defined chondral lesions, less than $2 \mathrm{~cm}^{2}$, of Outerbridge grade II-III, treated via arthroscopic RFC. Exclusion criteria included diffuse articular cartilage damage, additional pathologies affecting the knee or subsequent further injuries or invasive procedures to the knee. Data was collected via a questionnaire assessing patient outcome.
\end{abstract}

Results: 35 patients met the inclusion criteria, 32 were successfully contacted. Male: female ratio was 16:16, with a mean age of 39.5 (range 19-60).84\% $(n=27)$ of patients experienced a significant reduction in pain (mean reduction of $51 \%,(95 \% \mathrm{Cl} 41 \%$ to $61 \%), p<0.001)$ lasting until the time of study (median of 21 months, range 9 to 31 months). There was no correlation between change in symptoms and cause, site or grade of chondral lesion. There was no significant change to Cincinnati sports activity pre and post RFC. Pre-operative instability symptoms did not significantly improve following RFC. Satisfaction with treatment was in direct correlation with pain relief achieved.

Conclusions: Our study adds to current literature by suggesting short term improvements to pain purely following the use of RFC on isolated chondral lesions.

Keywords: Chondroplasty; Chondral lesions; Arthroscopy

\section{Introduction}

Partial thickness chondral defects are a common occurrence, being found in $60 \%$ of knee arthroscopies [1]. If left untreated they are known to worsen in size and symptoms, with swelling, delamination and fibrillation of the articular cartilage reducing its ability to deal with load $[2,3]$. Chondroplasty is a recognised treatment for pain associated with chondral damage [4]. Radiofrequency Energy Chondroplasty (RFC) is increasing in use following greater understanding of its underlying cellular effects [5-7]. RFC has been shown to give an improved outcome, both macroscopically and microscopically, when compared to mechanical chondroplasty, with decreased lesion fibrillation, reoccurrence and progression, and purportedly improved cartilage microstructure [5,7-9]. The long term sequelae and clinical outcomes of RFC have been poorly demonstrated. A recent literature review by Kosy et al. supported its use, demonstrating improved mechanical stability, with decreased pain and improved functional scores when comparing RFC to mechanical chondroplasty, but highlighted the lack of clinical evidence demonstrating long term outcome or risks [10].

We conducted a retrospective study on patients undergoing RFC of isolated chondral defects in the knee to assess post-operative pain relief and return to function. This paper highlights the short-term clinical outcomes.

We hypothesised significant short term improvement to pain following RFC of isolated chondral lesions of the knee.

\section{Materials and Methods}

Retrospective analysis was performed on all patients who underwent regional arthroscopic RFC between 2009 and 2012. Operative notes and arthroscopic images were analysed for study inclusion.

\section{Inclusion criteria}

- Arthroscopy of the knee performed between January 2009 and June 2012

- All ages

- 1 to 2 defined chondral lesions, each less than $2 \mathrm{~cm}^{2}$ of Outerbridge grade II-III, treated via RFC.

- 1 defined chondral lesion less than $2 \mathrm{~cm}^{2}$ of Outerbridge grade II-III treated via RFC, in the presence of one defined chondral lesion less than $2 \mathrm{~cm}^{2}$ of Outerbridge grade 4, edges annealed with RFC.

- Size of lesion was determined via analysis of intra-operative arthroscopic photos comparing lesion to probe size.

\section{Exclusion criteria}

- Additional pathology identified within the knee by diagnostic arthroscopy or pre-operative MRI, such as meniscal tears, cruciate damage or a collateral sprain.

- 1 to 2 chondral lesions each larger than $2 \mathrm{~cm}^{2}$

${ }^{*}$ Corresponding author: Timothy Woodacre, The Knee Unit, Princess Elizabeth Orthopaedic Centre, Royal Devon and Exeter Hospital, Barrack Road, Exeter, Devon, England, UK, Tel: +447824393322; E-mail: timwoodacre@yahoo.co.uk

Received January 22, 2014; Accepted March 26, 2014; Published April 04, 2014

Citation: Woodacre T, Thomas A, Mandalia VI (2014) Pain Relief Following Radio Frequency Energy Chondroplasty for Isolated Chondral Lesions of the Knee. J Arthritis 3: 125. doi:10.4172/2167-7921.1000125

Copyright: (c) 2014 Woodacre T, et al. This is an open-access article distributed under the terms of the Creative Commons Attribution License, which permits unrestricted use, distribution, and reproduction in any medium, provided the original author and source are credited. 
- 3 or more chondral lesions regardless of size

- Chondral lesions of Outerbridge grade 4 not fulfilling inclusion criteria

- Chondral lesions treated via alternative methods (for example, mechanical chondroplasty or micro fracture)

Diffuse articular cartilage damage

- Subsequent further injury or invasive procedure (repeat surgery or artificial synovial fluid injection) to the identified knee. Patients undergoing further targeted physiotherapy were not excluded.

\section{Operative technique}

Standard antero-medial and antero-lateral arthroscopy portals were used in all cases. Diagnostic arthroscopy of all compartments was performed to ascertain all underlying pathology. All cases were irrigated with normal saline at room temperature and employed suction to increase irrigation flow during RFC, reducing the potential risk of thermal necrosis. Bipolar radiofrequency electrical therapy utilising Arthrocare Sports Medicine Wand systems was employed in all cases. The specific technique for the use of the RFC probe was as described by Kosy et al. [10] A single pass of the RFC probe to each specific area of each lesion was employed to achieve a stable rim. Further passes were limited to regions not already treated as multiple passes of the same area have been shown not to improve results when actively in use the probe was kept continually moving to reduce point heating and the potential for local damage [10].

\section{Post-operative management}

All patients were initially given a standard post-operative physiotherapy regime, involving range-of-motion, proprioception and quadriceps resistance exercises. All were followed up in an orthopaedic surgeon-led clinic.

\section{Data collection}

Patients were sent retrospective questionnaires with objective and subjective scoring of change in pain following RFC.

\section{Data analysis}

Change in outcome pre and post RFC was analysed via Wilcoxon statistical analysis to determine significance.

\section{Results}

3174 patients underwent knee arthroscopy by the Royal Devon and Exeter NHS trust between January 2009 and June 2012. 218 patients underwent arthroscopic chondroplasty. 36 patients were suitable for inclusion within the study, with the majority of patients excluded due to the presence of additional pathology within the knee (principally meniscal tears) or the articular damage being too large and diffuse. 2 patients were excluded due to receiving additional treatment post RFC ( 2 artificial synovial fluid injections). Of the suitable 36 patients, 32 were successfully contacted to participate in the study. Male: Female ratio was 16: 16 . Mean age at time of surgery was 39.5 , range 19-60.

\section{Symptoms}

$100 \%$ of patients $(n=32)$ presented with pain within their knee. $41 \%(n=13)$ reported pre-operative feelings of knee instability. Only $38 \%(n=5)$ of patients experiencing pre-operative instability reported complete resolution of instability post-RFC (Figure 1).

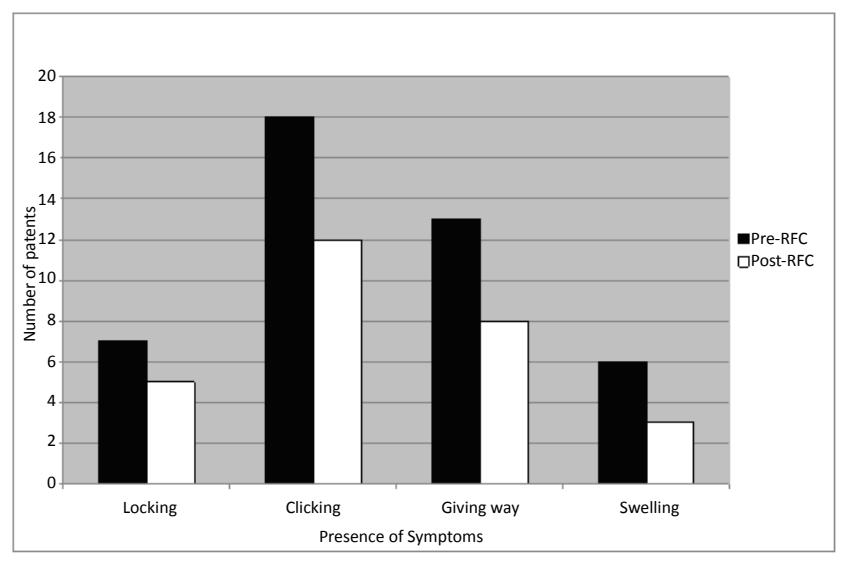

Figure 1: Pre and Post RFC Symptoms in Addition to Pain.

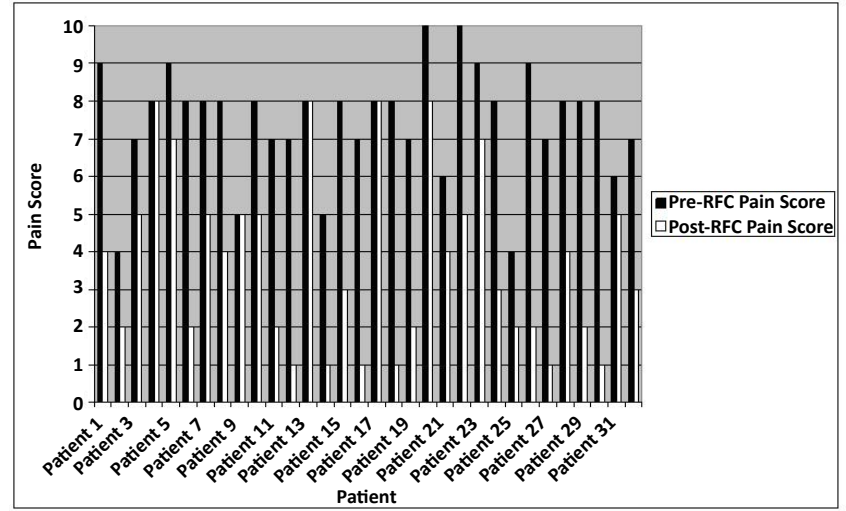

Figure 2: Relative Pain Scores Per Patient Pre and Post RFC.

\section{Pain control}

A visual pain analogue scale out of 10 was utilised to compare patients' pre-operative pain and their current levels of pain at the time of the study.

$13 \%(n=4)$ reported no change to their pain, $3 \%(n=1)$ reported an improvement in pain of only 1 out of 10 following RFC (Figures 2 and 3$)$.

$84 \%(n=27)$ of patients reported significant persistent improvement in pain of at least 2 out of 10 (Figure 4), correlating to at least a $20 \%$ persistent reduction in pain per patient (Figure 5); $\mathrm{p}=0.000003$ (Wilcoxon signed rank test).

Mean reduction in pain in all patients following RFC was by $51 \%$ ( $95 \%$ CI $41 \%$ to $61 \%$ ), $\mathrm{p}<0.001$ (Figure 6).

\section{Pain in relation to cause, location and grade of lesion}

$31 \%(n=10)$ of patients suffered knee pain following a defined precipitating event to their knee, suggesting chondral damage secondary to trauma. $69 \%(\mathrm{n}=22)$ of patients suffered knee pain without a defined precipitating event, suggesting chondral damage of a degenerative nature. There was no significant correlation between the cause of the lesion and pre-operative pain or pain relief achieved following RFC.

$28 \%(\mathrm{n}=9)$ of patients suffered chondral defects upon their patella 
Citation: Woodacre T, Thomas A, Mandalia VI (2014) Pain Relief Following Radio Frequency Energy Chondroplasty for Isolated Chondral Lesions of the Knee. J Arthritis 3: 125. doi:10.4172/2167-7921.1000125

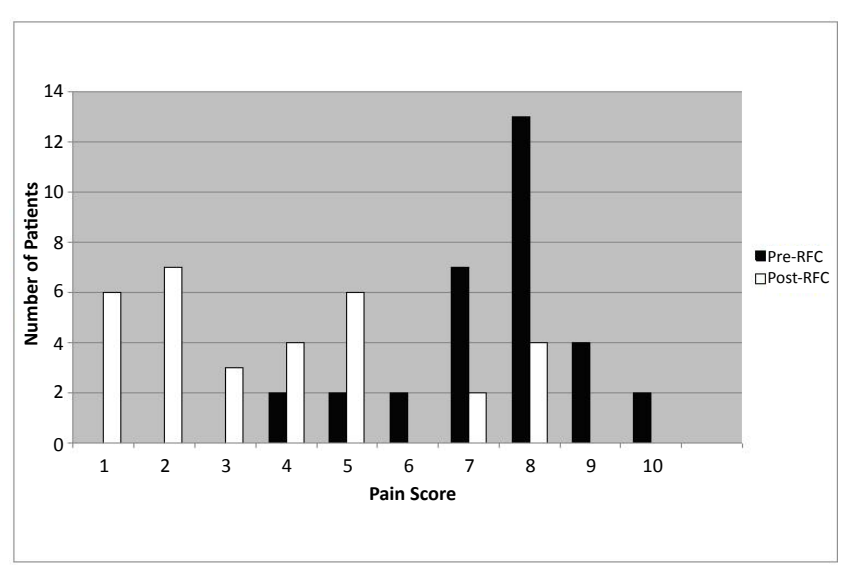

Figure 3: Overall Relative Pain Scores Pre and Post RFC.

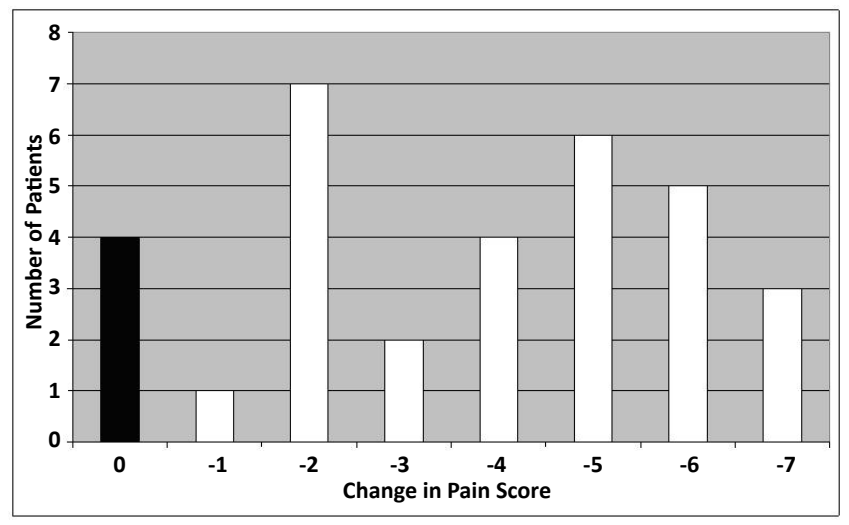

Figure 4: Change in Pain Score Following RFC.

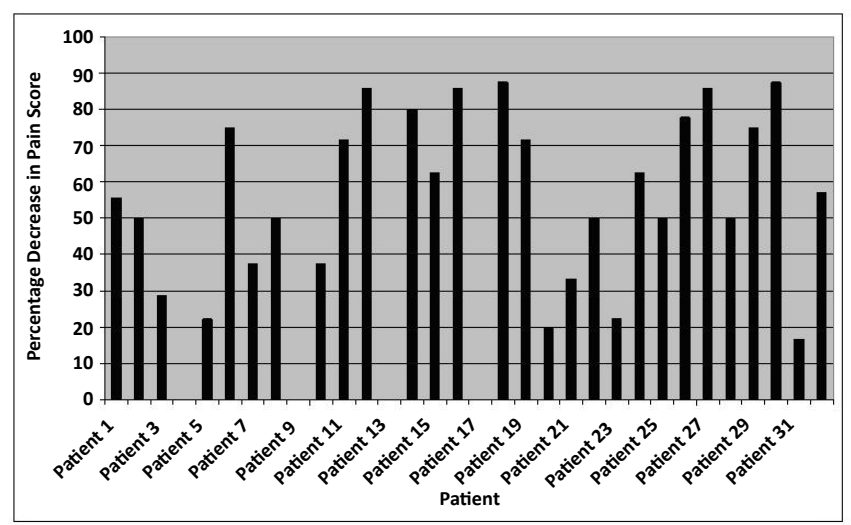

Figure 5: Percentage Decrease in Pain Score Per Patient Following RFC.

in isolation. A further $50 \%(n=16)$ of patients suffered chondral defects on their patella in combination with other sites. Therefore in total $78 \%$ $(n=25)$ of patients suffered defects upon the articular surface of their patella.

$16 \%(n=5)$ of patients suffered chondral defects on their medial femoral condyle in isolation. A further $31 \%(n=10)$ of patients suffered chondral defects on their medial femoral condyle in combination with other sites. Therefore in total $47 \%(n=15)$ of patients suffered chondral

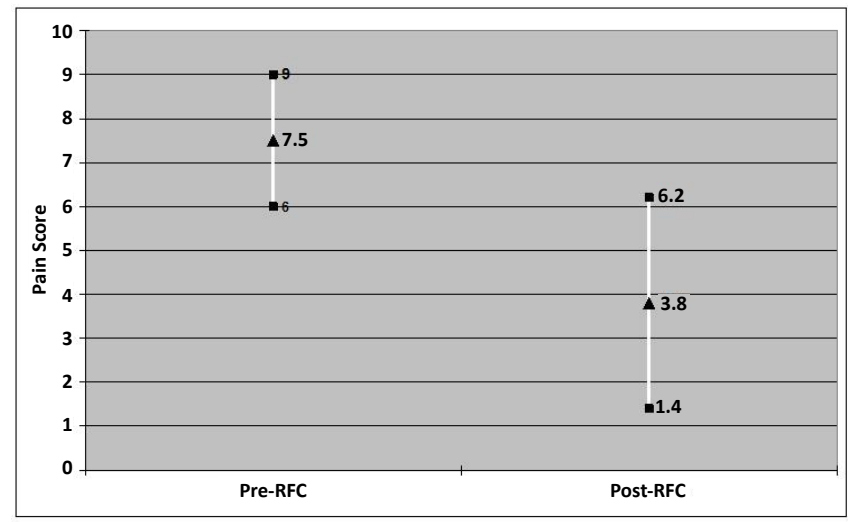

Figure 6: Combined Pain Scores Pre and Post RFC; Mean +/- 1 Standard Deviation.

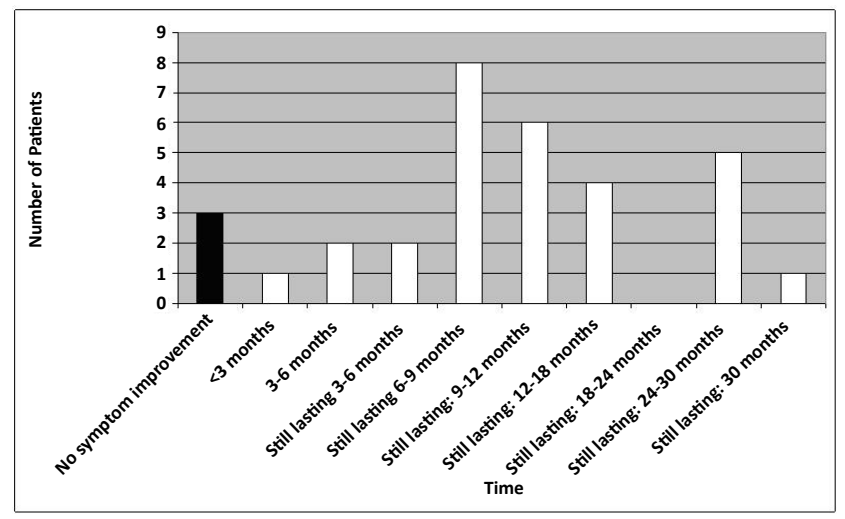

Figure 7: Duration of Maximal Symptom Improvement Following RFC.

defects upon their medial femoral condyle.

4 patients suffered one grade II-III lesion in the presence if one grade IV lesion. The remaining 28 patients suffered one to two grade II-III lesions.

The distribution and grade of lesions relative to the change in pain following RFC are summarised in Table 1. There was no significant correlation between location or grade of lesion and reduction in pain following RFC.

\section{Duration of symptom relief}

- Median time to follow-up post RFC was 11 months (range 3 to 39 months)

At the time of study:

- $91 \%(n=29)$ of patients were still experiencing improvement to their symptoms (one greater than those who were experiencing improvement purely to their pain).

- $81 \%(n=26)$ of patients were still experiencing maximal improvement to their symptoms (Figure 7).

- $9 \%(n=3)$ of patients were still experiencing some symptom relief, but had noticed a deterioration to their maximal symptom improvement. This deterioration occurred within 6 months of undergoing RFC. 
Citation: Woodacre T, Thomas A, Mandalia VI (2014) Pain Relief Following Radio Frequency Energy Chondroplasty for Isolated Chondral Lesions of the Knee. J Arthritis 3: 125. doi:10.4172/2167-7921.1000125

Page 4 of 6

\begin{tabular}{|c|c|c|c|c|c|c|c|c|c|c|c|}
\hline \multirow[t]{2}{*}{ Site of $1^{\text {st }}$ lesion } & \multicolumn{4}{|c|}{ Grade of 1st lesion } & \multirow[t]{2}{*}{ Site of concurrent $2^{\text {nd }}$ lesion } & \multicolumn{4}{|c|}{ Grade of $2^{\text {nd }}$ lesion } & \multirow[t]{2}{*}{ No. of patients } & \multirow[t]{2}{*}{ Mean change in pain } \\
\hline & 1 & 2 & 3 & 4 & & 1 & 2 & 3 & 4 & & \\
\hline \multirow[t]{13}{*}{ Patella } & $\mathrm{x}$ & & & & Med. tibial plateau & & $x$ & & & 1 & $0 \%$ \\
\hline & & $\mathrm{X}$ & & & & & & & & 3 & $30 \%$ \\
\hline & & $\mathrm{X}$ & & & Trochlea & & $X$ & & & 1 & $75 \%$ \\
\hline & & $\mathrm{X}$ & & & & & & & $X$ & 1 & $71 \%$ \\
\hline & & $\mathrm{X}$ & & & Med. femoral condyle & & $\mathrm{x}$ & & & 4 & $77 \%$ \\
\hline & & $\mathrm{X}$ & & & & & & $\mathrm{X}$ & & 2 & $39 \%$ \\
\hline & & & $\mathrm{X}$ & & & & & & & 6 & $47 \%$ \\
\hline & & & $\mathrm{X}$ & & Trochlea & & & $\mathrm{X}$ & & 1 & $33 \%$ \\
\hline & & & $\mathrm{X}$ & & & & & & $\mathrm{X}$ & 1 & $86 \%$ \\
\hline & & & $\mathrm{X}$ & & Med. femoral condyle & & $\mathrm{X}$ & & & 2 & $36 \%$ \\
\hline & & & $\mathrm{X}$ & & & & & $\mathrm{X}$ & & 1 & $86 \%$ \\
\hline & & & $\mathrm{X}$ & & & & & & $\mathrm{X}$ & 1 & $75 \%$ \\
\hline & & & & $\mathrm{X}$ & Trochlea & & $x$ & & & 1 & $50 \%$ \\
\hline Med. femoral condyle & & & $\mathrm{X}$ & & & & & & & 5 & $46 \%$ \\
\hline Lat. tibial plateau & & & $x$ & & & & & & & 1 & $0 \%$ \\
\hline Trochlea & & & $\mathrm{X}$ & & Lat. femoral condyle & & & $\mathrm{X}$ & & 1 & $57 \%$ \\
\hline
\end{tabular}

Table 1: Site and ascending grade of chondral lesion relative to change in pain post RFC.

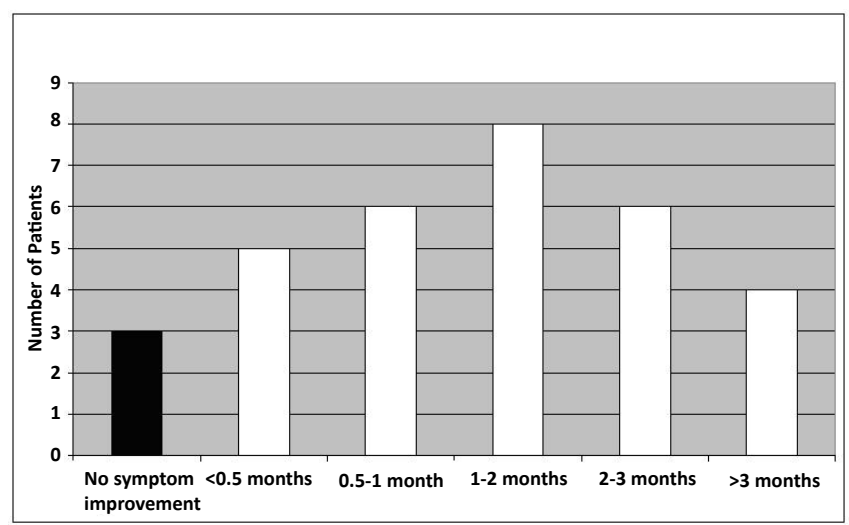

Figure 8: Time Following RFC to Maximal Relief of Symptoms.

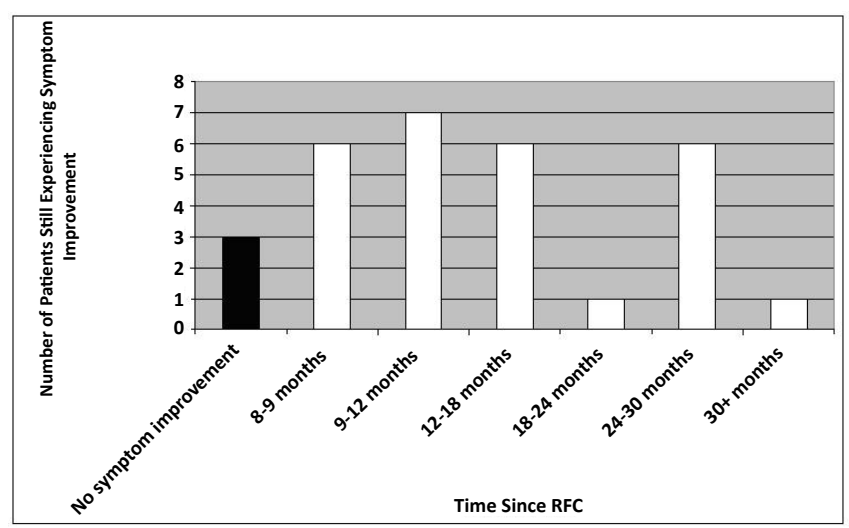

Figure 9: Duration of Symptom Improvement Following RFC

- $9 \%(n=3)$ of patients had experienced no improvement to symptoms.

- Median maximal improvement in symptoms occurred 1-2 months after RFC (Figure 8).
When one excludes the 2 patients who's time to study follow-up was less than 6 months, $90 \%(n=27)$ of patients were still experiencing some (if not maximal) symptom relief at least 6 months following their arthroscopy, median of 13.5 months (Figure 9).

\section{Activity levels}

Only $47 \%(n=15)$ of patients completed pre and post RFC Cincinnati sports activity scales (Figure 10). There was no significant overall change to activity pre and post RFC. Mean pre RFC score was 6.9 (95\% CI 5.2 to 8.6 ) mean post RFC score was 6.7 (95\% CI 5.5 to 7.9 ) with a mean change of -0.2 (2.5 to -2.9$), \mathrm{p}=0.67$.

\section{Satisfaction with treatment}

$50 \%(n=16)$ of patients were satisfied and $34 \%(n=11)$ were very satisfied with the outcome of their operation. $16 \%(n=5)$ were not satisfied. Satisfaction correlated directly to percentage reduction in pain with $60 \%(n=3)$ of patients unsatisfied with the procedure experiencing no pain relief following RFC.

\section{Discussion}

A limited number of recent studies have purported the short term benefit of RFC in conjunction with other treatments. Spahn et al. [1] conducted a prospective study in 2008 examining medial femoral condyle chondroplasty in conjunction with medial meniscectomy. This demonstrated improved pain, function and quality of life scores of up to a year when RFC was utilised. A randomised prospective study by Barber et al. [4] in 2006 demonstrated significantly improved pain and outcome scores in patients 19 months post either mechanical chondroplasty, or mechanical chondroplasty combined with RFC, but with no significant difference in outcomes between the two. One study by Owens et al. [11] in 2002 compared RFC directly to mechanical chondroplasty. This demonstrated improved outcomes at one and two years in the RFC treatment group, yet was restricted to the treatment of grade 2-3 chondral lesions of the patella in female patients.

Concerns have existed regarding the potential long term side effects of RFC, most notably localised AVN of the articular cartilage from exposure to high local temperatures. Chu et al. [6] and Voss et al. [8] demonstrated chondrocyte death from protracted or repeated exposures to temperature exceeding $50^{\circ} \mathrm{C}$. This is not thought to be representative 
I take part 4-7 days a week in sports involving:

1. Jumping, hard pivoting sutting (e. g. rugby, basketball, volleyball or gymnastic)

2. Running, twisting, turning (e. g. tennis, squash, badminton, hockey, skiing. golf, rock climbing).

3. No running, twisting or jumping (e. g. cuycling, swimming, rowing).

I take part 1-3 days a week in sports involving:

4. Jumping, hard pivoting cutting (e. g. rugby, basketball, volleyball or gymnastic)

5. Running, twisting, turning (e. g. tennis, squash, badminton, hockey, skiing, golf, rock climbing).

6. No running, twisting or jumping (e. g. cycling swimming, rowing).

I take part 1-3 days a month in sports involving:

7. Jumping, hard pivotng cutting (e.g. rugby, basketball, volleyball or gymnastic)

8. Running, twisting, turning (e. g. tennis, squash, badminton, hockey, skiing, golf, rock climbing).

9. No running, twisting or jumping (e.g. cycling, swimming, rowing).

I do not take part in sports

10. I perfprm activities of daily living without problems.

11. I have moderate problems with activities of daily living.

12. I have severe problems with activities of daily living, on crutches, with full disability.

Figure 10: Cincinnati Sports Activity Scale.

of arthroscopic RFC however due to the short time period of exposure to the probe and the use of irrigated saline. Limited clinical data exists to exclude AVN as a long term risk following RFC, however Barber and Iwasko [4] demonstrated no clinical or radiological evidence of AVN at one year. Likewise, no published clinical data exists to demonstrate the long-term clinical benefits of RFC and whether pain relief is sustained and further surgical intervention prevented or delayed. Voloshin et al. [8] demonstrated at least partial filling of chondral lesions in over $50 \%$ of patients up to 30 months following RFC however the underlying reason behind this is unknown as is long term patient clinical outcome.

Our study showed a statistically significant reduction in pain following RFC, with a mean reduction in pain of $51 \%(\mathrm{p}<0.001)$.

Our study suggests that maximal pain relief following RFC can take a few months (median=1-2) to develop. Those patients who do experience pain relief experience it for greater than 6 months (all patients who experienced pain relief were still gaining benefit at the time of the study, median time from operation to study=13.5 months). This concurs with the work of Spahn et al. [1] and Owens et al. [11] demonstrating significant short-term clinical benefit from RFC.

Strict inclusion criteria ensured that our study assessed purely the outcome of RFC on isolated chondral lesions, without the confounding factors of additional articular, meniscal or ligament pathology or additional treatment modalities [12]. This severely limited the population size of the study, reducing inclusion numbers from 3174 total knee arthroscopies and 218 arthroscopies involving chondroplasty completed over 3 years, to 36 patients who matched the criteria. However this strengthened the relevance of the clinical result, ensuring that clinical outcome correlated directly to the effects of RFC. Despite the strict inclusion criteria, patient numbers were still of sufficient magnitude to demonstrate statistical significance.

Our study demonstrated no significant correlation between the cause, site or grade of chondral defect and pain relief following RFC.

Assessment of patient activity levels via the Cincinnati sports activity scale was limited due to low patient participation. Cincinnati scoring demonstrated no significant change to activity levels pre and post RFC. 
Citation: Woodacre T, Thomas A, Mandalia VI (2014) Pain Relief Following Radio Frequency Energy Chondroplasty for Isolated Chondral Lesions of the Knee. J Arthritis 3: 125. doi:10.4172/2167-7921.1000125

Page 6 of 6

Despite the lack of significance, this suggests that the improvement to pain scores achieved following RFC was not a consequence of decreased patient activity.

The study was conducted retrospectively and thus the time following surgery to data collection was variable (median 11 months, range 4-33 months) with no control (such as arthroscopic washout alone) used for comparison. The retrospective nature of the study precluded the use of standardised pre-operative patient scoring by means such as KOOS. The focus of the study however was on pain relief following RFC, rather than overall multi-factorial return to function

2 patients were excluded due to receiving additional artificial synovial fluid injections to their knee post RFC. This would suggest that an additional 2 patient's received no or minimal symptom relief following RFC. This would not however affect the statistical significance of the improvement in pain score pre and post RFC $(\mathrm{p}<0.001)$.

Our study supports current literature and suggests short term pain relief following the use of RFC on chondral lesions in the knee. Due to its strict inclusion criteria it adds to current literature by suggesting pain relief purely following the effects of RFC on grade II-III lesions, excluding all other knee pathology and treatments. No current literature exists demonstrating long term clinical outcomes; therefore extending the study would aid knowledge in this field.

\section{References}

1. Spahn G, Kahl E, Möckley T, Hoffmann GO, Klinger HM (2008) Arthroscopic knee chondroplasty using a bipolar radiofrequency-based device compared to mechanical shaver: Results of a prospective, randomised, controlled study. Knee Surg Sports Traumatol Arthrosc 16: 565-573.
2. Edwards RB III, Lu Y, Markel MD (2002) The basic science of thermally assisted chondroplasty. Clin Sports Med 21: 619-647.

3. Brittberg M, Winalski CS (2003) Evaluation of cartilage injuries and repair. J Bone Joint Surg Am 85: 58-69.

4. Barber FA, Iwasko NG (2006) Treatment of grade III femoral chondral lesions: Mechanical chondroplasty versus monopolar radiofrequency probe. Arthroscopy 22: 312-1317.

5. Meyer ML, Lu Y, Markel MD (2005) Effects of radiofrequency energy on human chondromalacic cartilage: An assessment of insulation material properties. IEEE Trans Biomed Eng 52: 702-710.

6. Voss JR, Lu Y, Edwards RB, Bogdanske JJ, Markel MD (2006) Effects of thermal energy on chondrocyte viability. Am J Vet Res 67: 1708-1712.

7. Yasura K, Nakagawa Y, Kobayashi M, Kuroki H, Nakamura T (2006) Mechanical and biochemical effect of monopolar radiofrequency energy on human articular cartilage: An in vitro study. Am J Sports Med 34: 1322-1327.

8. Voloshin I, Morse KR, Allred CD, Bissell SA, Maloney MD, et al. (2007) Arthroscopic evaluation of radiofrequency chondroplasty of the knee. Am J Sports Med 35: 1702-1707.

9. Edwards RB, Lu Y, Cole BJ, Muir P, Markel MD (2008) Comparison of radiofrequency treatment and mechanical debridement of fibrillated cartilage in an equine model. Vet Comp Orthop Traumatol 21: 41-48.

10. Kosy JD, Schranz PJ, Toms AD, Eyres KS, Mandalia VI (2011) The Use of Radiofrequency Energy for Arthroscopic Chondroplasty in the Knee. Arthroscopy 27: 695-703.

11. Owens BD, Stickles BJ, Balikian P, Busconi BD (2002) Prospective analysis of radiofrequency versus mechanical debridement of isolated patellar chondral lesions. Arthroscopy 18: 151-155.

12. Chu CR, Kaplan LD, Fu FH, Crossett LS, Studer RK (2004) Recovery of articular cartilage metabolism following thermal stress is facilitated by IGF-1 and JNK inhibitor. Am J Sports Med 32: 191-196. 